\title{
APS 2-ID Microprobes: Status and Future Plans
}

Barry Lai ${ }^{1, *}$, Dana Capatina ${ }^{1}$, Chris Roehrig ${ }^{1}$, Olga Antipova ${ }^{1}$, Zhonghou Cai $^{1}$, Si Chen ${ }^{1}$, Junjing Deng ${ }^{1}$.

1. X-ray Science Division, Advanced Photon Source, Argonne National Laboratory, Argonne, IL

* Corresponding author, blai@anl.gov

Beamline 2-ID-D/E at the APS provide advanced scanning microprobe capabilities to diverse user communities such as materials science, energy science, environmental science, life science. Applications include, for instance, measuring microelectronics defects in 3D, local structure and electronics state in advanced photovoltaic materials, elemental content and oxidation state of aerosol particles, exogenous and endogenous metal distribution in cells and tissues. Station 2-ID-D currently hosts two instruments: an XRF/XANES microprobe with 200-nm resolution [1], and a fast ptychography instrument called the Velociprobe [2]. Station 2-ID-E hosts an XRF microprobe with tomographic capability. All three scanning probes utilize Fresnel zone plates as the microfocusing optics.

Even though both 2-ID-D and 2-ID-E stations operate full time, they share a collinear beam from the undulator source, thus changing energy or selecting degree of coherence separately is difficult. Also, beam stability has been an ongoing issue. Therefore the entire 2-ID beamline will be canted in 2019 to provide independent operation of the 2-ID-D and 2-ID-E station. Since the canting requires replacement of most beamline components, it is expected that the installation and commissioning activities may take up to 9 months. The canted 2-ID beamline is designed to achieve highly coherent and stable beams for the scanning probe operation in the 2-ID-D and 2-ID-E station (Fig. 1). Essentially both branchlines employ a mirror-first approach to further increase the separation between the two beamlines. The first mirrors also serve to reduce thermal load to downstream optics and reject higher harmonics. The mirrors are bendable and may be used to refocus the source horizontally onto a beam defining aperture (BDA) which acts as a stable secondary source and provides spatial filtering. The $\mathrm{x}$-ray intensity and coherence can be selected by varying the BDA opening. The 2-ID-D branchline, covering $4.5-30 \mathrm{keV}$, will be served by a horizontal double crystal monochromator (H-DCM) located upstream of the BDA. The 2-ID-E branchline, covering $4.5-20 \mathrm{keV}$, will be served by a vertical double multilayers monochromator (DMM) upstream of the BDA and a Kohzu double crystal monochromator (DCM) inside the 2-ID-E station.

In the longer term the APS Upgrade with the multibend achromat (MBA) lattice, currently projected to start in mid-2022 and continue through most of 2023, promises to increase the source brightness hence coherence flux by two orders of magnitude. To take advantage of the higher flux without significantly increasing the radiation dose, the scanning speed of all 2-ID microprobes needs to be increased also. It is envisioned that the XRF/XANES microprobe currently at 2-ID-D will be replaced by an upgraded Bionanoprobe (BNP-II) [3], specially designed to provide 10-nm resolution for samples under cryogenic environment. The 2D/3D XRF microprobe in 2-ID-E will be upgraded with a pair of precisely figured KB mirror to attain 50-100 nm spatial resolution. KB mirrors not only has high reflectivity, but also are achromatic which enables true micro-XAS capability and allows a single set of optics to cover the entire $5-20 \mathrm{keV}$ regime, simplifying operation. Higher intensity is particularly needed in order to render XRF tomography a practical technique, where long scan time had in the past led to sample drift or deterioration. With the enhancement, 2-ID-E will be able to provide a high-throughput versatile microprobe, bridging the length scale between other nanoprobes (10's of nm) and microprobes (micron) at the APS. While the Bionanoprobe (BNP-II) will provide very high spatial resolution, the 2-ID-E microprobe will continue to 
provide easy access, flexible sample size and geometry, and accommodating environments such as high temperature, controlled humidity, cryogenic, hazardous gases.

References:

[1] Z. Cai, B. Lai, W. Yun, P. Ilinski, D. Legnini, J. Maser, W. Rodrigues, AIP Proc. 507 (X-Ray Microscopy VI), 472-477 (2000).

[2] Jeffrey A. Klug et al., this proceeding.

[3] Si Chen et al., J. Synchrotron. Rad. 21, 66-75 (2014).

[4] This research used resources of the Advanced Photon Source, a U.S. Department of Energy (DOE) Office of Science User Facility operated for the DOE Office of Science by Argonne National Laboratory under Contract No. DE-AC02-06CH1 1357.

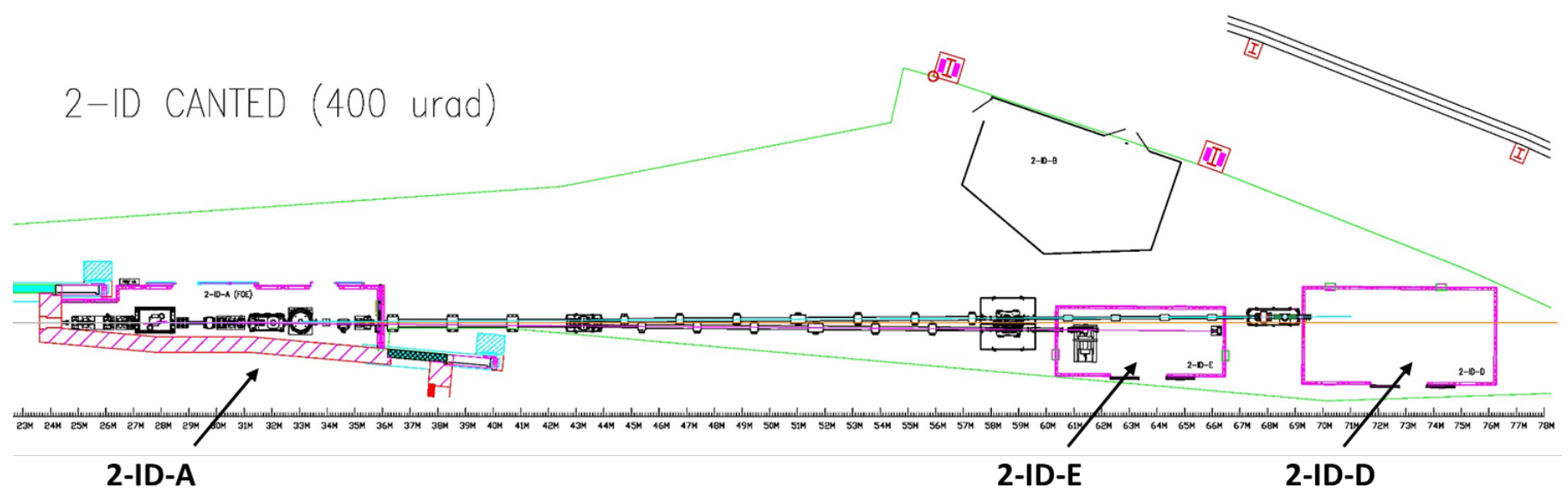

Figure 1. Layout of the canted 2-ID beamline, enabling independent operation of the 2-ID-D and 2-ID-E endstation. 